\title{
Treatment options after sorafenib failure in patients with hepatocellular carcinoma
}

\author{
Imane El Dika' and Ghassan K. Abou-Alfa, ${ }^{1,2}$ \\ 'Memorial Sloan Kettering Cancer Center, ${ }^{2}$ Weill Cornell Medical College, New York, NY, USA
}

Second line therapy after failure of sorafenib continues to be under study. Prognosis of hepatocellular carcinoma is measured in months, with median overall survival reaching 10.7 months with sorafenib. Because of the modest net benefit sorafenib has contributed, and rising incidence of hepatocellular carcinoma in the world, continued efforts are ongoing to look for efficient upfront, second line, or combination therapies. Herein we review the most relevant to date published literature on treatment options beyond sorafenib, reported studies, ongoing investigational efforts, and possibilities for future studies in advanced hepatocellular carcinoma. (Clin Mol Hepatol 2017;23:273-279)

Keywords: Hepatocellular carcinoma; Antiangiogenic therapy; MET-inhibitors; Immunotherapy

\section{INTRODUCTION}

Sorafenib remains the only effective first line systemic treatment, with no other approved therapies in the first line yet. Lenvatinib, another antiangiogenic agent, was just shown to be non-inferior to sorafenib. After almost a decade of disappointing results of studies in both first and second line setting, recent data showed improved outcome with regorafenib after progression on sorafenib. The success outcome of regorafenib as an agent by itself or to its sequential use after sorafenib, warrants acknowledgment and a lot of exploration at the biological and molecular level. In the meantime, immunotherapy and c-MET inhibition are continued to be investigated; with the expected results from the immunotherapy standpoint, positioning of these drugs is the next challenge for oncologists. Herein we will discuss the recent results and anticipated ones from a second line perspective.

\section{TARGETING ANGIOGENESIS}

Angiogenesis is initiated by destabilization of existing microvasculature, which leads to vascular hyper-permeability, remodeling of the extracellular matrix, and endothelial cell activation. Activated endothelial cells proliferate, migrate, and form new vessels. ${ }^{1}$ In hepatocellular carcinoma (HCC), a net excess of angiogenic factors produced by tumor cells, vascular endothelial cells, and immune cells leads to the activation and recruitment of endothelial cells and pericytes ${ }^{2}$ The plasma concentration of vascular endothelial growth factor (VEGF), angio-

\footnotetext{
Abbreviations:

ADI-PEG 20, PEGylated arginine deiminase; AFP, a-fetoprotein; ANGPT, angiopoietin; $\mathrm{Cl}$, confidence interval; CTLA, cytotoxic T-lymphocyte-associated protein; EGF, epidermal growth factor; FGF, fibroblast growth factor; FU, Fluorouracil; HCC, hepatocellular carcinoma; $\mathrm{HCV}$, hepatitis $\mathrm{C}$ virus; $\mathrm{HGF}$, hepatocyte growth factor; IGF, Insulin growth factor; OS, Overall survival; ORR, Objective response rate; PDGF, platelet-derived growth factor; PDL, Programmed Death Ligand; PFS, Progression free survival; TGF, transforming growth factor; TIE-2, tyrosine kinase with immunoglobulin-like and EGF-like domains 2; TKI, Tyrosine kinase inhibitor; TTP, Time to progression; VEGF, vascular endothelial growth factor
}

Corresponding author: Ghassan K. Abou-Alfa

Memorial Sloan Kettering Cancer Center, 300 East 66th Street, New York, New York 10065, USA

Tel: +1-646-888-4184, Fax: +1-646-888-4255

E-mail: abou-alg@mskcc.org

http://orcid.org/0000-0002-1522-8054 
poietin-2 (Ang2), and platelet-derived growth factor (PDGF)-B was found to be increased in patients with HCC compared to cirrhotic patients. $^{3}$

In addition to VEGF, the angiopoietin (ANGPT) family of ligands, ANGPT1 and ANGPT2, bind to the endothelial cell membrane receptor tyrosine kinase TIE2 and contribute to tumor angiogenesis. Concurrent or sequential targeting of the VEGF and angiopoietin pathways is of interest because the strategy could improve efficacy without increasing toxicity. ${ }^{4}$ The most common proposed mechanism of resistance is related to the increased level of tumor hypoxia caused by antiangiogenic therapy. ${ }^{5}$ For example, inhibition of the VEGF pathway leads to resumption of tumor angiogenesis through upregulation of fibroblast growth factor (FGF)2, interleukin 8, and ANGPT2. Hence, targeting alternative angiogenic pathways is a potential strategy to deal with this mechanism. Another aspect of resistance to antiangiogenic drugs is the potentially reversible (epigenetic) nature of resistance. ${ }^{6}$ This change in tyrosine kinase inhibitor (TKI) profile has implications for second-line use of alternate antiangiogenic drugs-e.g., when switching VEGFR TKI in renal cell carcinoma, and more recently in HCC.

Other angiogenic factors potentially involved in liver cancer are placental growth factor (PIGF), basic fibroblast growth factor (bFGF), transforming growth factor (TGF)- $\alpha$, TGF- $\beta$, hepatocyte growth factor (HGF), epidermal growth factor (EGF), IL4, IL-6, and IL-8.

In view of the successful outcome of sorafenib, an oral multi kinase inhibitor that inhibits VEGFR1-3, PDGFR- $\alpha$, PDGFR- $\beta, c-$ KIT, Raf-1 and BRAF, angiogenesis became of the most appealing targets to explore in HCC. Many antiangiogenic agents were explored after progression on sorafenib.

In the phase III multicenter, double-blind, placebo-controlled trial (SHARP) 602 patients with advanced HCC and no previous systemic treatment were randomly assigned to receive either sorafenib (at a dose of $400 \mathrm{mg}$ twice daily) or placebo. Sorafenib showed an improvement in median survival to 10.7 months compared to 7.9 months in the placebo group, $(\mathrm{HR}=0.69 ; P<0.001) .{ }^{8}$ Of note, sorafenib was reported to be well tolerated among the Korean patients, and survival was in line with the results from the SHARP study. ${ }^{9}$ While benefit from sorafenib is observed across patients regardless of disease stage and etiology, subgroup analyses of the phase $\mathrm{II}^{10}$ and III studies of sorafenib in HCC have implied an improved benefit of sorafenib in patients with hepatitis C virus (HCV)-induced HCC versus other causes." A randomized phase III trial from the Asia-Pacific region ${ }^{12}$ involving patients with disease induced mainly by hepatitis B showed that, compared with placebo, sorafenib had a statistically significant survival advantage, but not to the same magnitude as in the SHARP trial (6.5 vs. 4.2 months; $P=.014)$. More recently, a metaanalysis of the phase III randomized trials that demonstrated correlation between sorafenib effect and hepatitis status was reported. It showed improved overall survival (OS) for sorafenib in patients who are both HBV negative and HCV positive (HR, 20.27; 95\% $\mathrm{Cl}, 20.46$ to 20.06). Median unadjusted survival is 12.6 months for sorafenib and 10.2 months for "other" treatments in this subgroup..$^{13}$ The preferential activity of sorafenib in HCV induced HCC, if real, might be due to high RAF kinase activity driven by HCV core protein-1, in this subgroup. ${ }^{14}$

Regorafenib a TKI that targets tyrosine kinase with immunoglobulin-like and EGF-like domains 2 (TIE-2), FGFR, c-kit, and Ret in addition to VEGF, PDGFR, and RAF-MEK-ERK, was evaluated after progression on sorafenib in a randomized, international, multicenter, phase III trial (RESORCE). ${ }^{15}$ Study included 573 patients with documented radiological progression during sorafenib treatment who were randomly $(2: 1)$ assigned to regorafenib or placebo. Patients were $B C L C$ stage $B$ or $C$, Child-Pugh $A$ liver function, they must have tolerated prior sorafenib (defined by $\geq 400 \mathrm{mg}$ daily for at least 20 of the 28 days before discontinuation), and have received their last sorafenib dose within 10 weeks of randomization (median 0.9 month). Median duration of sorafenib therapy was 7.8 months. Study showed clinically and statistically significant improvement in OS (10.6 vs 7.8 months; $P<0.001)$ and progression free survival (PFS) (3.1 vs 1.5 months; $P<0.001)$. Objective responses were achieved by RECIST 1.1, survival benefit was observed across subgroup populations, including Asian vs non-Asian, hepatitis B or C, and regardless of $\alpha$-fetoprotein (AFP) level. An unplanned analysis revealed a combined OS on both drugs that reached 26 months. ${ }^{16}$ Benefit from regorafenib despite being valid, is still an ambiguity. The question is how regorafenib could overcome sorafenib resistance, despite being very similar. It is unknown whether a surge in VEGF and angiogenesis after failure of sorafenib, involvement of other pathways, or altogether can explain its efficacy. A small study investigated the effect of sorafenib beyond first progression, with modest OS benefit $(P=0.012) .^{17}$ Suppressing angiogenesis beyond progression may have survival benefit on its own; add to it targeting other pathways with regorafenib. Preclinical data suggests that sorafenib treated cell lines express insulin growth factor-1 (IGF-1) ${ }^{18}$, so far 
regorafenib spectrum of action does not include IGF pathway. Both sorafenib and regorafenib were shown to be antagonized in vitro by platelet growth factors, suggesting a common escape mechanism. ${ }^{19}$ Is regorafenib exclusively efficient after sorafenib failure, in other words, does sorafenib set the stage for it, is a crucial question. It is unknown yet if this effect would be similarly observed in the second line regardless of the first line therapy, namely immunotherapy, or perhaps in the first line. Molecular biomarkers and clinical correlations studies are needed to help elucidate mechanisms of action and resistance, and guide us for potential combination therapies. Ramucirumab, a recombinant IgG1 monoclonal antibody and VEGF receptor-2 antagonist was assessed in advanced HCC following first-line therapy with sorafenib, in a randomized phase III trial. Median OS for the ramucirumab group was 9.2 months (95\% Cl 8.0-10.6) versus 7.6 months (6.0-9.3) for the placebo group (HR 0.87 [95\% Cl 0.72-1.05]; $P=0.14$ )..$^{20}$ In this study, a significant percentage of patients had elevated AFP above $400 \mathrm{ng} / \mathrm{ml}$. In this subgroup, median OS was 7.8 months (95\% Cl 5.8-9.3) for the ramucirumab group versus 4.2 months (3.7-4.8) for the placebo group.

Molecular classification has shown a unique subclass of HCC with elevated baseline AFP and enriched with growth signaling kinases, such as FGFR3, FGFR4, and IGF2 and its receptor, which might increase VEGF/VEGFR-2 pathway activity. Elevated AFP has been associated with elevated VEGFR expression, increased angiogenesis, and poor prognosis in hepatocellular carcinoma. ${ }^{21}$ Currently, a study of ramucirumab versus placebo in patients with elevated AFP is ongoing (REACH-2, NCT02435433).

Cediranib, another pan-VEGFR tyrosine kinase inhibitor, was looked at in a single center phase II study. Eighty nine per cent of patients received prior systemic therapy and 59\% received prior sorafenib. Cediranib at $30 \mathrm{mg}$ daily resulted in an estimated 3-month-PFS rate of 77\% [60\%, 99\%]. Median PFS was $5.3[3.5,9.7]$ months, and median OS was 11.7 [7.5-13.6] months. ${ }^{22}$ Previous study showed high toxicity and 5.8 months OS when cediranib was given at $45 \mathrm{mg}$ daily. ${ }^{23}$ Cediranib was not further explored in larger conclusion generating studies.

Brivanib is a selective dual inhibitor of VEGF and FGF receptors, both implicated in HCC tumorigenesis and angiogenesis. Phase II study including 46 patients revealed a tumor response rate of $4.3 \%$; the disease control rate was $45.7 \%$ and median OS was 9.79 months. ${ }^{24}$ Following to these results, a phase III study randomized 395 patients with advanced HCC who progressed on/after or were intolerant to sorafenib to receive brivanib $800 \mathrm{mg}$ orally once per day or placebo. Median OS was 9.4 months for brivanib and 8.2 months for placebo (HR, $0.89 ; 95.8 \% \mathrm{Cl}, 0.69$ to $1.15 ; P=0.3307)^{25}$, showing no added benefit from targeting FGF in this particular study.

In agreement with the antiangiogenesis boom, lenvatinib, an oral inhibitor of VEGFR1-3, FGFR1-4, PDGFR- $\alpha$, RET, and KIT, was explored and is the last agent to mark and eventually reach the angiogenic ceiling. A phase 2 single-arm study including 46 patients was conducted at sites across Japan and Korea. $34 \%$ of patients received prior systemic therapy, and only $13 \%$ received prior sorafenib. The median time to progression (TTP) was 7.4 months [95\% Cl: 5.5-9.4]. 17 patients (37\%) had partial response and 19 patients (41\%) had stable disease (objective response rate (ORR): $37 \%$; disease control rate (DCR): $78 \%$ ). Median OS was 18.7 months ( $95 \%$ Cl: 12.7 25.1). The most common any-grade adverse events were hypertension, palmar-plantar erythrodysesthesia syndrome, decreased appetite, and proteinuria. ${ }^{26}$ The impressive results led to the evaluation of lenvatinib in the first line. Indeed, the open-label, phase III trial (NCT01761266), of lenvatinib versus sorafenib, the last to be reported in the multi-kinase antiangiogenic category, has met its primary endpoint of non-inferiority. ${ }^{27} 954$ patients were enrolled, OS was 13.6 months with lenvatinib compared to 12.3 months with sorafenib, HR 0.92 (0.79-1.06). Lenvatinib achieved statistically significant and clinically meaningful improvements in PFS, TTP, and ORR.

\section{TARGETING MET}

Accumulating evidence has established the role of the MET receptor tyrosine kinase, encoded by the MET proto-oncogene, in tumor development and metastatic progression. Binding of HGF to MET activates primarily the RAS-MAPK and PI3K-AKT signaling pathways. MET is overexpressed in HCC compared with non-tumor liver tissue, with higher MET expression linked to poor prognosis.

Tivantinib is a selective, oral, small-molecule MET inhibitor that preferentially inhibits growth and induces apoptosis in human tumor cell lines expressing MET. A multicenter, randomized, double-blind, placebo-controlled, phase 2 trial was conducted in patients who had progressed on one previous systemic therapy. First impression results were negative with median PFS of 1.5 months $(95 \% \mathrm{Cl} 1.4-2.7)$ in the tivantinib group versus 1.4 months $(95 \% \mathrm{Cl} 1.4-1.5)$ in the placebo 
group (HR 0.67, 95\% Cl 0.44-1.04; $P=0.06$ ), and no difference in median OS. However, the subgroup of patients with MET overexpression showed an improvement in median OS from 3.8 (2.1-6.8) to 7.2 months ( $95 \% \mathrm{Cl} 3.9-14.6$ ) (HR 0.38, $95 \% \mathrm{Cl} 0.18-0.81 ; P=0.01) .{ }^{28}$ This doubling in survival led to the phase III trial (NCT01755767) of tivantinib after sorafenib exclusively in patients with MET-high tumors, defined by $\geq 2+$ in $\geq 50 \%$ of tumor cells by immunohistochemistry. The completed study did not meet its primary endpoint, and tivantinib failed to improve OS or PFS compared to placebo as per recently reported data. ${ }^{29}$ Median OS $(95 \% \mathrm{Cl})$ was 8.4 months (6.8-10.0) in tivantinib vs 9.1 months (7.3-10.4) in placebo, $H R=0.97(0.75-1.25), P=0.81$. Definition of MET overexpression has not been validated so far and might not be as simple as that, taking into consideration intratumoral heterogeneity. At the same time, cabozantinib, an oral potent inhibitor of MET, RET, VEGFR2, and TIE-2, is being evaluated in "all comers" advanced HCC, regardless of MET expression, after failure of prior sorafenib therapy (CELESTIAL). Investigators chose another approach taking advantage of the multiple pathways involved in HCC and the multi targeting ability of the drug. Preclinical experiences have shown that sorafenib resistant mouse models exhibit high levels of activated $\mathrm{MET}^{30}$, cabozantinib has also activity against $A X L-1^{31}$, part of the TAM receptor tyrosine kinase subfamily, involved in tumor growth and migration. In the phase II randomized discontinuation trial, 41 patients who had received at least one prior systemic therapy received cabozantinib $100 \mathrm{mg}$ daily vs placebo. Prior sorafenib use was $51 \%$. PFS reached 4.2 months. ${ }^{32}$ The ongoing phase 3 randomized, double-blind, controlled study includes patients regardless of MET expression. ${ }^{33}$ The primary endpoint is OS and secondary endpoints are PFS and ORR by RECIST 1.1. The study met its primary endpoint of OS as per recent press release. Final results will be presented at a scientific meeting.

\section{IMMUNOTHERAPY}

Preclinical data have indicated that several immunologic mechanisms contribute to $\mathrm{HCC}$ development and growth while impairing effective host antitumor immune surveillance. ${ }^{34}$ Little is known about programmed death-ligand 1 (PD-L1) expression in $\mathrm{HCC}$; in a retrospective review of 240 patients with HCC, it was shown that PDL-1 was constitutively expressed in HCC tumor specimens, in a focal or scattered manner. Patients with higher expression of PD-L1 by immunohistochemistry had a significantly poorer prognosis than patients with lower expression. ${ }^{35}$ In another study 217 HCC patients were evaluated for PDL-1 expression, it was observed in $17 \%$ of tumors and ranged from $1 \%$ to $30 \%$ of positive cells. PD-L1 expression by neoplastic or intratumoral inflammatory cells in HCC was significantly associated with common markers of tumor aggressiveness (high serum AFP levels, satellite nodules, macrovascular invasion, microvascular invasion, and poor differentiation) ${ }^{36}$ Several studies are ongoing to evaluate the role of immune checkpoint blockade in HCC, both upfront and after sorafenib, looking into potential role of prior therapy exposure and the ideal positioning of these agents.

A phase 2 study of tremelimumab in patients with advanced HCC with HCV-related cirrhosis with majority of patients failing prior sorafenib established promising activity. Of 17 patients, 3 (17.6\%) achieved a partial response and 10 patients (58.8\%) had stable disease. The DCR was $76.4 \%$, and clinical benefit was $>12$ months in approximately one-third of patients. The median time to disease progression was 6.5 months, which is favorable compared with historical controls for this population. Interestingly, clearance of hepatitis C virus was observed among participants, and most common treatment-related adverse event was elevation in transaminases. ${ }^{37}$ Segal, et al. reported the preliminary results of MEDI4736 (durvalumab), a human IgG1 monoclonal antibody to PD-L1, found to be tolerable, with lower rates of hepatotoxicity than observed with cytotoxic T-lymphocyte-associated protein (CTLA)-4 blockade in patients with HCC. Of 19 evaluable patients, there were no responders according to RECIST1.1, although $21 \%$ of patients achieved disease control at 12 weeks. ${ }^{38}$ Tremelimumab and MEDI4736 are being evaluated each as monotherapy and in combination in patients who failed or were intolerant to sorafenib (NCT02519348).

Nivolumab, a fully human IgG4 monoclonal antibody to PD1, was tested in an HCC-specific phase 1/2 trial. Efficacy was encouraging, with 2 complete responses noted and an overall objective response rate of $19 \%$ by RECIST. The OS rate at 12 months was $62 \%(95 \% \mathrm{Cl}, 42 \%-76 \%){ }^{39}$ Final results were recently reported ${ }^{40}, 262$ patients were treated (48 patients in the dose-escalation phase and 214 in the dose-expansion phase). During dose escalation, nivolumab showed a manageable safety profile, including acceptable tolerability. $25 \%$ of patients had grade 3/4 treatment-related adverse events. Nivolumab 3 $\mathrm{mg} / \mathrm{kg}$ was chosen for dose expansion. The objective response 
rate was $20 \%(95 \% \mathrm{Cl} 15-26)$ in patients treated with nivolumab $3 \mathrm{mg} / \mathrm{kg}$ in the dose-expansion phase and 15\% (95\% Cl 6-28) in the dose-escalation phase. Responses were observed across etiologies, regardless of etiology, tumor PD-L1 expression and sorafenib exposure. Interestingly, preclinical data suggests that low levels of pERK are associated with sorafenib resistance. Mouse and human HCC samples expressing low pERK showed strong inflammatory infiltrating cells and enrichment of intratumoral CD8+ cytotoxic $T$ lymphocytes that express PD-1 ${ }^{41}$, suggesting that sorafenib resistance may confer better response to immunotherapy, which remains a theory to be verified. Currently, nivolumab is being compared to sorafenib in the first line setting (NCT02576509); while pembrolizumab is being evaluated in patients who failed prior systemic therapy (NCT02658019). These studies might inform us if there is a major difference in immunotherapy activity based on time of administration. Other innovative approaches have been proposed to enhance efficacy of immunotherapy and manipulate the cancer and its microenvironment, such as combining embolization and radiation therapy to immunotherapy, with the idea of stimulating immunogenicity. Safety and efficacy of Y90 radio-embolization with nivolumab is being evaluated currently (NCT02837029).

Other targets are being evaluated in the second line therapy after sorafenib, such as PEGylated arginine deiminase (ADI-PEG 20), a systemic arginine deprivation agent.

Human HCC cells require exogenous arginine for growth, and it has been shown that they are deficient of argininosuccinate synthetase, the urea cycle enzyme required to catalyze the conversion of citrulline to arginine, thus, auxotrophic for arginine. These observations indicated that depletion of circulating arginine might have a potential anti-cancer effect (ADI-PEG 20), was demonstrated to be potentially active and safe in a phase I/II study ${ }^{42}$ including 35 patients with HCC. A randomized phase II study was conducted in Taiwan ${ }^{43}$ and included 71 patients, with $89 \%$ having failed prior therapies. 4 months' disease control rates were $15 \%$ for $160 \mathrm{lU} / \mathrm{m}^{2}$ group, and $16 \%$ for $320 \mathrm{IU} / \mathrm{m}^{2}$ group. Median OS was 7.4 months, and survival appeared to correlate well with the duration of plasma arginine depletion. Another phase II study evaluated ADI-PEG 20 in Asian patients with advanced HCC, 44\% had failed previous therapies. There were no objective responders. The DCR and the median OS of the intent-to-treat population were 31.0\% (95\% confidence interval (Cl): 20.5-43.1) and 7.3 (95\% Cl: 4.79.9) months respectively. ${ }^{44} 80$ Caucasian patients with unre- sectable and metastatic HCC were included in a phase II study, mean survival was 15.8 months. ${ }^{45} \mathrm{~A}$ phase III randomized clinical trial evaluated ADI-PEG 20 in patients who failed prior systemic therapy. Median OS was 7.8 months vs 7.4 months for placebo $(P=0.884, \mathrm{HR}=1.022$ (95\% Cl: $0.847,1.233))$ and median PFS 2.6 vs $2.6(P=0.075, \mathrm{HR}=1.175$ (95\% Cl: 0.964 , 1.432)). Interestingly, patients with arginine depletion for $>8$ weeks had a median OS of 12.3 months compared to 7.3 months ( $P=0.0032)$ for $\leq 4$ weeks. Similarly, patients with citrulline increase for $>8$ weeks had a median OS of 11.6 months, compared to 3.5 months $(P<0.0001)$ for $\leq 4$ weeks. ${ }^{46}$

ADI-PEG 20 and 5-fluorouracil (FU) both inhibit thymidylate synthase (TS). ${ }^{47}$ Arginine deprivation was shown to have additive effect with 5-FU in inhibiting $\mathrm{HCC}$ in a xenograft model. ${ }^{48}$ ADI-PEG 20 demonstrated synergy with oxaliplatin in inhibiting HCC growth in a xenograft model. ${ }^{49}$ An ongoing phase I, open label, dose-escalation study is exploring the combination of FOLFOX and ADI-PEG 20 in HCC and other GI malignancies (NCT02102022). After reaching the MTD, expansion cohort is initiated to further assess toxicity and obtain preliminary estimates of efficacy.

\section{CONCLUSION}

Sorafenib is not the only approved therapy in advanced HCC anymore. Benefit remains concrete across etiologies despite likely inferior role in HBV induced HCC. Lenvatinib was shown to be non-inferior to sorafenib and another approved first-line therapy is expected in 2018. Regorafenib and nivolumab are now approved second line therapies in HCC after progression on sorafenib. Cabozantinib is expected to become another second-line option as well. Results from CheckMate-459, now approaching full accrual, are awaited and might change our first-line choice. The enrichment in HCC treatment landscape was long awaited. Lining up or possibly combining these therapies and choosing wisely among them will be the next challenge. Correlative studies are needed to better elucidate clinical and molecular biomarkers, resistance pathways, and inform future treatment strategies.

\section{Authors' contribution}

Both authors contributed in the outline of the work and drafting the article. Ghassan Abou-Alfa provided critical revision of the article and final approval to be published. 


\section{Conflicts of Interest}

The authors have no conflicts to disclose.

\section{REFERENCES}

1. Dvorak HF. Vascular permeability factor/vascular endothelial growth factor: a critical cytokine in tumor angiogenesis and a potential target for diagnosis and therapy. J Clin Oncol 2002;20:4368-4380.

2. Zhu AX, Duda DG, Sahani DV, Jain RK. HCC and angiogenesis: possible targets and future directions. Nat Rev Clin Oncol 2011;8:292-301.

3. Mas VR, Maluf DG, Archer KJ, Yanek KC, Fisher RA. Angiogenesis soluble factors as hepatocellular carcinoma noninvasive markers for monitoring hepatitis $C$ virus cirrhotic patients awaiting liver transplantation. Transplantation 2007;84:1262-1271.

4. Jayson GC, Kerbel R, Ellis LM, Harris AL. Antiangiogenic therapy in oncology: current status and future directions. Lancet 2016;388:518-529.

5. Bergers G, Hanahan D. Modes of resistance to anti-angiogenic therapy. Nat Rev Cancer 2008;8:592-603.

6. Tang TC, Man S, Xu P, Francia G, Hashimoto K, Emmenegger U, et al. Development of a Resistance-like Phenotype to Sorafenib by Human Hepatocellular Carcinoma Cells Is Reversible and Can Be Delayed by Metronomic UFT Chemotherapy. Neoplasia 2010;12:928940

7. Semela D, Dufour JF. Angiogenesis and hepatocellular carcinoma. J hepatol 2004;41:864-880.

8. Llovet JM, Ricci S, Mazzaferro V, Hilgard P, Gane E, Blanc JF, et al. Sorafenib in advanced hepatocellular carcinoma. N Engl J Med 2008;359:378-390.

9. Kim DY, Kim HJ, Han KH, Han SY, Heo J, Woo HY, et al. Real-life experience of sorafenib treatment for hepatocellular carcinoma in Korea: from GIDEON data. Cancer Res Treat 2016;48:1243-1252.

10. Abou-Alfa GK, Schwartz L, Ricci S, Amadori D, Santoro A, Figer A, et al. Phase II study of sorafenib in patients with advanced hepatocellular carcinoma. J Clin Oncol 2006;24:4293-4300.

11. Abou-Alfa GK. Selection of patients with hepatocellular carcinoma for sorafenib. J Natl Compr Canc Netw 2009;7:397-403.

12. Cheng AL, Kang YK, Chen Z, Tsao CJ, Qin S, Kim JS, et al. Efficacy and safety of sorafenib in patients in the Asia-Pacific region with advanced hepatocellular carcinoma: a phase III randomised, doubleblind, placebo-controlled trial. Lancet Oncol 2009;10:25-34.

13. Jackson R, Psarelli EE, Berhane S, Khan H, Johnson P. Impact of viral status on survival in patients receiving sorafenib for advanced hepatocellular cancer: a meta-analysis of randomized phase III trials. J Clin Oncol 2017;35:622-628.

14. Giambartolomei S, Covone F, Levrero M, Balsano C. Sustained activation of the Raf/MEK/Erk pathway in response to EGF in stable cell lines expressing the hepatitis C Virus (HCV) core protein. Oncogene
2001;20:2606-2610

15. Bruix J, Qin S, Merle P, Granito A, Huang YH, Bodoky G, et al. Regorafenib for patients with hepatocellular carcinoma who progressed on sorafenib treatment (RESORCE): a randomised, double-blind, placebo-controlled, phase 3 trial. Lancet 2017;389:56-66.

16. Bruix J, Merle P, Granito A, Huang YH, Bodoky G, Okosuka O, et al. Efficacy and safety of regorafenib versus placebo in patients with hepatocellular carcinoma (HCC) progressing on sorafenib: results of the international, randomized phase 3 RESORCE trial. [Abstract]. Ann Oncol 2016;27(Suppl 1):ii140-ii141.

17. Miyahara K, Nouso K, Morimoto Y, Takeuchi Y, Hagihara H, Kuwaki K, et al. Efficacy of sorafenib beyond first progression in patients with metastatic hepatocellular carcinoma. Hepatol Res 2014;44:296-301.

18. Tovar V, Cornella H, Moeini A, Vidal S, Hoshida Y, Sia D, et al. Tumour initiating cells and IGF/FGF signalling contribute to sorafenib resistance in hepatocellular carcinoma. Gut 2017;66:530-540.

19. D'Alessandro R, Refolo MG, Lippolis C, Giannuzzi G, Carella N, Messa $C$, et al. Antagonism of sorafenib and regorafenib actions by platelet factors in hepatocellular carcinoma cell lines. BMC cancer 2014;14:351

20. Zhu AX, Park JO, Ryoo BY, Yen CJ, Poon R, Pastorelli D, et al. Ramucirumab versus placebo as second-line treatment in patients with advanced hepatocellular carcinoma following first-line therapy with sorafenib (REACH): a randomised, double-blind, multicentre, phase 3 trial. Lancet Oncol 2015;16:859-870.

21. Yamashita T, Forgues $M$, Wang W, Kim JW, Ye Q, Jia H, et al. EpCAM and alpha-fetoprotein expression defines novel prognostic subtypes of hepatocellular carcinoma. Cancer Res 2008;68:1451-1461.

22. Zhu AX, Ancukiewicz M, Supko JG, Sahani DV, Blaszkowsky LS, Meyerhardt JA, et al. Efficacy, safety, pharmacokinetics, and biomarkers of cediranib monotherapy in advanced hepatocellular carcinoma: a phase II study. Clin Cancer Res 2013;19:1557-1566.

23. Alberts SR, Fitch TR, Kim GP, Morlan BW, Dakhil SR, Gross HM, et al. Cediranib (AZD2171) in patients with advanced hepatocellular carcinoma: a phase II North Central Cancer Treatment Group Clinical Trial. Am J Clin Oncol 2012;35:329-333.

24. Finn RS, Kang YK, Mulcahy M, Polite BN, Lim HY, Walters I, et al. Phase II, open-label study of brivanib as second-line therapy in patients with advanced hepatocellular carcinoma. Clin Cancer Res 2012;18:2090-2098.

25. Llovet JM, Decaens T, Raoul JL, Boucher E, Kudo M, Chang C, et al. Brivanib in patients with advanced hepatocellular carcinoma who were intolerant to sorafenib or for whom sorafenib failed: results from the randomized phase III BRISK-PS study. J Clin Oncol 2013;31:3509-3516

26. Ikeda K, Kudo M, Kawazoe S, Osaki Y, Ikeda M, Okusaka T, et al. Phase 2 study of lenvatinib in patients with advanced hepatocellular carcinoma. J Gastroenterol 2017;52:512-519. 
27. Cheng AL, Finn RS, Qin S, Han KH, Ikeda K, Piscaglia F, et al. Phase III trial of lenvatinib (LEN) vs sorafenib (SOR) in first-line treatment of patients (pts) with unresectable hepatocellular carcinoma (uHCC). J Clin Oncol 2017;35(Suppl):4001.

28. Santoro A, Rimassa L, Borbath I, Daniele B, Salvagni S, Van Laethem $J \mathrm{~L}$, et al. Tivantinib for second-line treatment of advanced hepatocellular carcinoma: a randomised, placebo-controlled phase 2 study. Lancet Oncol 2013;14:55-63.

29. Rimassa L, Assenat E, Peck-Radosavljevic M, Zagonel V, Pracht M, Caremoli ER, et al. Second-line tivantinib (ARQ 197) vs placebo in patients (Pts) with MET-high hepatocellular carcinoma (HCC): Results of the METIV-HCC phase III trial. J Clin Oncol 2017;35(Suppl):4000.

30. Xiang Q, Chen W, Ren M, Wang J, Zhang H, Deng DY, et al. Cabozantinib suppresses tumor growth and metastasis in hepatocellular carcinoma by a dual blockade of VEGFR2 and MET. Clin Cancer Res 2014;20:2959-2970.

31. Zhou L, Liu XD, Sun M, Zhang X, German P, Bai $S$, et al. Targeting MET and $A X L$ overcomes resistance to sunitinib therapy in renal cell carcinoma. Oncogene 2016;35:2687-2697.

32. Cohn AL, Kelley RK, Yang TS, Su WC, Vrslype C, Ramies DA, et al. Activity of cabozantinib (XL184) in hepatocellular carcinoma: Results from a phase II randomized discontinuation trial (RDT). J Clin Oncol 2012;30(Suppl):261.

33. Abou-Alfa GK, Cheng AL, Meyer T, El-Khoueiry AB, Ikeda M, Chun $H G$, et al.Phase 3 randomized, double-blind, controlled study of cabozantinib (XL184) versus placebo in subjects with hepatocelIular carcinoma who have received prior sorafenib (CELESTIAL; NCT01908426). J Clin Oncol 2014;32(Suppl):TPS4150.

34. Harding JJ, El Dika I, Abou-Alfa GK. Immunotherapy in hepatocellular carcinoma: Primed to make a difference? Cancer 2016;122:367-377.

35. Gao Q, Wang XY, Qiu SJ, Yamato I, Sho M, Nakajima Y, et al. Overexpression of PD-L1 significantly associates with tumor aggressiveness and postoperative recurrence in human hepatocellular carcinoma. Clin Cancer Res 2009;15:971-979.

36. Calderaro J, Rousseau B, Amaddeo G, Mercey M, Charpy C, Costentin $C$, et al. Programmed death ligand 1 expression in hepatocellular carcinoma: Relationship with clinical and pathological features. Hepatology 2016;64:2038-2046.

37. Sangro B, Gomez-Martin C, de la Mata M, Iñarrairaegui M, Garralda E, Barrera P, et al. A clinical trial of CTLA-4 blockade with tremelimumab in patients with hepatocellular carcinoma and chronic hepatitis C. J Hepatol 2013;59:81-88.

38. Segal NH, Hamid O, Hwu W, Massard C, Butler M, Antonia S, et al. A phase I multi-arm dose-expansion stydy of the anti-programmed cell death-ligand-1 (PD-L1) antibody medi4736: preliminary data. Ann Oncol 2014;25(Suppl 4):iv365.

39. El-Khoueiry AB, Melero I, Crocenzi TS, Welling TH, Yau TC, Ye W, et al. Phase I/II safety and antitumor activity of nivolumab in patients with advanced hepatocellular carcinoma (HCC): CA209-040. J Clin Oncol 2015;33(Suppl):Iba101.

40. El-Khoueiry AB, Sangro B, Yau T, Crocenzi TS, Kudo M, Hsu C, et al. Nivolumab in patients with advanced hepatocellular carcinoma (CheckMate 040): an open-label, non-comparative, phase 1/2 dose escalation and expansion trial. Lancet 2017;389:2492-2502.

41. Chen J, Ji T, Zhao J, Li G, Zhang J, Jin R, et al. Sorafenib-resistant hepatocellular carcinoma stratified by phosphorylated ERK activates PD-1 immune checkpoint. Oncotarget 2016;7:41274-41284.

42. Delman KA, Brown TD, Thomas M, Ensor CM, Holtsberg FW, Bomalaski JS, et al. Phase I/II trial of pegylated arginine deiminase (ADI-PEG20) in unresectable hepatocellular carcinoma. J Clin Oncol 2015;23(Suppl):4139.

43. Chen LT, Chen PJ, Chan Y, Sheen IS, Yang TS, Wang TE, et al. A randomized phase II study of pegylated arginine deiminase (ADI-PEG 20) in advanced hepatocellular carcinoma (HCC) in Taiwan. [Abstract] Gastrointestinal Cancers Symposium 2009;255:2009.

44. Yang TS, Lu SN, Chao Y, Sheen IS, Lin CC, Wang TE, et al. A randomised phase II study of pegylated arginine deiminase (ADI-PEG $20)$ in Asian advanced hepatocellular carcinoma patients. Br J Cancer 2010;103:954-960.

45. Glazer ES, Piccirillo M, Albino V, Di Giacomo R, Palaia R, Mastro $A A$, et al. Phase II study of pegylated arginine deiminase for nonresectable and metastatic hepatocellular carcinoma. J Clin Oncol 2010;28:2220-2226.

46. Abou-Alfa GK, Qin S, Ryoo BY, Lu SN, Yen CJ, Feng YH, et al. Phase III randomized study of second line ADI-peg 20 (A) plus best supportive care versus placebo $(\mathrm{P})$ plus best supportive care in patients (pts) with advanced hepatocellular carcinoma (HCC). J Clin Oncol 2016;34(Suppl);4017.

47. Allen MD, Luong P, Hudson C, Leyton J, Delage B, Ghazaly E, et al. Prognostic and therapeutic impact of argininosuccinate synthetase 1 control in bladder cancer as monitored longitudinally by PET imaging. Cancer Res 2014;74:896-907.

48. Cheng PN, Lam TL, Lam WM, Tsui SM, Cheng AW, Lo WH, et al. Pegylated recombinant human arginase (rhArg-peg5,000mw) inhibits the in vitro and in vivo proliferation of human hepatocellular carcinoma through arginine depletion. Cancer Res 2007;67:309-317.

49. Chow AK, Ng L, Sing Li H, Cheng CW, Lam CS, Yau TC, et al. Antitumor efficacy of a recombinant human arginase in human hepatocellular carcinoma. Curr Cancer Drug Targets 2012;12:1233-1243. 